\title{
Brightest cluster galaxies in the extended GMRT radio halo cluster sample
}

\section{Radio properties and cluster dynamics}

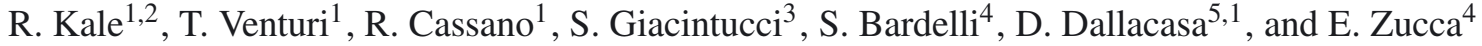 \\ ${ }^{1}$ INAF-Istituto di Radioastronomia, via Gobetti 101, 40129 Bologna, Italy \\ e-mail: tventuri@ira.inaf.it \\ 2 National Centre for Radio Astrophysics, Tata Institute of Fundamental Research, Post Bag 3, Ganeshkind, Pune 411007, India \\ 3 Department of Astronomy, University of Maryland, College Park, MD 20742-2421, USA \\ 4 INAF-Osservatorio Astronomico, via Ranzani 1, 40126 Bologna, Italy \\ 5 Dipartimento di Fisica e Astronomia, Universitá di Bologna, via Ranzani 1, 40126 Bologna, Italy
}

Received 17 April 2015 / Accepted 17 June 2015

\section{ABSTRACT}

\begin{abstract}
Aims. First-ranked galaxies in clusters, usually referred to as brightest cluster galaxies (BCGs), show exceptional properties over the whole electromagnetic spectrum. They are the most massive elliptical galaxies and show the highest probability to be radio loud. Moreover, their special location at the centres of galaxy clusters raises the question of the role of the environment in shaping their radio properties. In the attempt to separate the effect of the galaxy mass and of the environment on their statistical radio properties, we investigate the possible dependence of the occurrence of radio loudness and of the fractional radio luminosity function on the dynamical state of the hosting cluster.

Methods. We studied the radio properties of the BCGs in the Extended GMRT Radio Halo Survey (EGRHS), which consists of 65 clusters in the redshift range $0.2-0.4$, with X-ray luminosity $L_{\mathrm{X}} \geq 5 \times 10^{44} \mathrm{erg} \mathrm{s}^{-1}$, and quantitative information on their dynamical state from high-quality Chandra imaging. We obtained a statistical sample of 59 BCGs, which we divided into two classes, depending on whether the dynamical state of the host cluster was merging $(\mathrm{M})$ or relaxed $(\mathrm{R})$.

Results. Of the 59 BCGs, 28 are radio loud and 31 are radio quiet. The radio-loud sources are favourably located in relaxed clusters $(71 \%)$, while the reverse is true for the radio-quiet BCGs, which are mostly located in merging systems $(81 \%)$. The fractional radio luminosity function for the BCGs in merging and relaxed clusters is different, and it is considerably higher for BCGs in relaxed clusters, where the total fraction of radio loudness reaches almost $90 \%$, to be compared to the $\sim 30 \%$ in merging clusters. For relaxed clusters, we found a positive correlation between the radio power of the BCGs and the strength of the cool core, consistent with previous studies on local samples.

Conclusions. Our study suggests that the radio loudness of the BCGs strongly depends on the cluster dynamics; their fraction is considerably higher in relaxed clusters. We compare our results with similar investigations and briefly discuss them in the framework of AGN feedback.
\end{abstract}

Key words. radio continuum: galaxies - galaxies: clusters: general - galaxies: active $-\mathrm{X}$-rays: galaxies: clusters

\section{Introduction}

First-ranked galaxies are the brightest and most massive galaxies in the Universe and inhabit the cores of galaxy clusters. Galaxies in this class are both elliptical and $\mathrm{cD}$ and are commonly referred to as brightest cluster galaxies (BCGs). They represent the bright end of the luminosity function of early-type galaxies, with a small luminosity dispersion around the mean value. They are located at a small distance from the peak of the thermal $\mathrm{X}$-ray emission from the intracluster medium (ICM) and have small velocity dispersions (Quintana \& Lawrie 1982).

As a result of their special location at the centres of the largest gravitationally bound structures in the Universe, BCGs have enjoyed special attention for a long time. Many of them exhibit exceptional properties, with emission in the UV and farinfrared (FIR) and also in $\mathrm{H}_{\alpha}$ lines, suggesting the presence of multiphase gas and ongoing star formation (e.g. O'Dea et al. 2008, 2010; Haarsma et al. 2010; Donahue et al. 2010; Edge et al. 2010; Liu et al. 2012).
BCGs are a special class also in radio wavelengths. A large fraction of them shows radio emission of nuclear origin. In a number of cases the radio emission extends well beyond the optical envelope to form extended radio jets, which bend in a $\mathrm{C}$ shape (wide-angle tail sources) as a result of galaxy motion and cluster weather (Burns 1998). A prototypical case is the radio galaxy 3C 465 at the centre of A 2634 (Eilek et al. 1984). Except for some remarkable cases, their radio power is either at the transition between galaxies of Faranoff-Riley class I and II (FRI and FRII respectively, Fanaroff \& Riley 1974) or below (Owen \& Laing 1989).

Over the past decade, our view and understanding of the properties of the central regions in galaxy clusters has improved thanks to the contribution of the X-ray observatories Chandra and XMM-Newton. The radiative cooling of the $\mathrm{X}$-ray emitting gas in cool-core clusters (Peterson \& Fabian 2006) requires some source of heating to balance the radiative losses, and the AGN activity associated with the BCG in those systems is the primary candidate to provide this energy (McNamara \& Nulsen 2007). The existence of aged radio 
plasma, detected at frequencies below $1 \mathrm{GHz}$ and associated with X-ray cavities in a number of rich and poor clusters, is interpreted as the signature of repeated radio outbursts from the BCG and strongly supports the AGN feedback picture (e.g. Clarke et al. 2005, 2009; Fabian et al. 2002; Giacintucci et al. 2011a; McNamara \& Nulsen 2012).

An important tool for investigating the nature of the radio loudness in elliptical galaxies is the fractional radio luminosity function (RLF), which is defined as the probability that an elliptical galaxy of a given optical magnitude hosts a radio galaxy with radio power above a threshold value. A number of studies show that the RLF strongly depends on the optical magnitude of the associated galaxy (e.g. Auriemma et al. 1977; Ledlow \& Owen 1996; Mauch \& Sadler 2007; Bardelli et al. 2010) and is higher for brighter absolute optical magnitudes. BCGs are by definition the brightest galaxies and show the highest probability to be radio loud. However, BCGs are special not only in terms of mass, but also because of their location at the cluster centre, and it is important to separate these two effects. Previous studies suggested that the fractional radio luminosity function is independent of the galaxy environment (rich clusters, groups, and field). On the other hand, Best et al. (2007) found that BCGs are more likely to be radio loud than other galaxies of similar mass, and this effect becomes stronger for galaxies with stellar mass $M<10^{11} M_{\text {Sun }}$, suggesting that their location at the cluster centre does play a role in their radio properties. The importance of the local environment was clear also from the work of Mittal et al. (2009), who studied the radio properties of the HIghest X-ray FLUx Galaxy Cluster Sample (HIFLUGCS, Reiprich \& Böhringer 2002) and found that radio loud BCGs are more abundant in cool-core clusters: their fraction increases from $45 \%$ in non-cool-core (NCC) to $100 \%$ in strong cool-core (SCC) systems, and their radio power shows a positive correlation with the cool-core strength.

In this context, and motivated by the importance of separating the effect of the galaxy mass from that of the local environment, we addressed the question of the radio properties of BCGs in connection with the dynamical status of the host cluster, with the aim of providing a complementary picture to previous literature studies. We used the Extended Giant Metrewave Radio Telescope (GMRT) Radio Halo Survey (EGRHS), which includes 65 clusters in the redshift interval $0.2 \leq z \leq 0.4$ observed at $610 \mathrm{MHz}$ (see Venturi et al. 2007, 2008, hereafter V07 and V08; Kale et al. 2013, 2015, hereafter K13 and K15). The main goal of the EGRHS was to investigate the origin of diffuse cluster-scale radio sources in galaxy clusters, namely radio halos, mini-halos, and relics. Thanks also to the results of the EGRHS, it is quite clear today that the origin of large diffuse emission in galaxy clusters is related to the cluster dynamical status (e.g. Brunetti \& Jones 2014, for a review). In particular, it has been statistically shown that giant radio halos are associated with merging galaxy clusters (V07, Cassano et al. 2010, hereafter C10), like radio relics (e.g. de Gasperin et al. 2014). On the other hand, radio mini-halos always surround a radio-active BCG at the centre of relaxed cool-core clusters (Giacintucci et al. 2014; ZuHone et al. 2013, K15). Diffuse cluster scale emission in galaxy clusters can thus be used as tracer of the cluster dynamics, together with the more direct probes supplied by X-ray imaging and analysis.

In this paper we present the radio properties and fractional radio luminosity function of the BCGs in the EGRHS and relate these quantities to the cluster dynamical status (merger versus relaxed), which we derived quantitatively using high-quality Chandra images. The paper is organized as follows. In Sect. 2 we report on the selection criteria for our BCG sample and provide an overview of the sample as a whole; the radio properties of the BCGs and the X-ray properties of the host clusters are presented in Sect. 3; in Sect. 4 we present the statistical radio properties of the BCGs and the dynamical properties of the host cluster, and we describe the method of deriving the fractional radio luminosity function. A discussion of our results and conclusions are given in Sects. 5 and 6, respectively.

We adopted a standard $\Lambda \mathrm{CDM}$ cosmology to convert observed quantitites into intrinsic ones $\left(H_{0}=70 \mathrm{~km} \mathrm{~s}^{-1} \mathrm{Mpc}^{-1}\right.$, $\Omega_{\mathrm{M}}=0.29$ ). The convention $S \propto v^{-\alpha}$ is used throughout the paper.

\section{BCG sample}

The sample of BCGs presented in this work was extracted from the EGRHS (V07, V08, K13, and K15), which consists of galaxy clusters selected from the ROSAT-ESO flux-limited X-ray (REFLEX) galaxy cluster catalogue (Böhringer et al. 2004) and from the extended ROSAT Brightest Cluster Sample (EBCS) catalogue (Ebeling et al. 1998, 2000) according to the following criteria:

$$
\begin{aligned}
& \text { - } L_{\mathrm{X}}(0.1-2.4 \mathrm{keV})>5 \times 10^{44} \mathrm{erg} \mathrm{s}^{-1} \\
& -0.2 \leq z \leq 0.4 ; \text { and } \\
& -\delta>-31^{\circ} \text {. }
\end{aligned}
$$

From the original cluster sample we removed A 689, whose $\mathrm{X}$-ray luminosity has recently been revised and is below our threshold (Giles et al. 2012), which left 65 clusters, whose BCGs were identified by visual inspection of the optical images. Where available, we used images from data release 7 (DR7) of the Sloan Digital Sky Survey (SDSS, Ahn et al. 2014), otherwise we used the red plate of the Digitized Sky Survey (DSS-2). To identify the BCGs, we searched for the brightest cluster member in the proximity of the X-ray surface brightness peak in the NASA Extragalactic Database (NED). Proprietary and archival Chandra and XMM-Newton X-ray images were used to this aim. Out of the full cluster sample, six clusters host two BCGs (see Sect. 2.1), while no obvious one was found in three clusters (see Sect. 2.2).

The final BCG sample includes 68 objects (in 62 clusters). Table 1 reports the galaxies used for our statistical analysis, while Table 2 lists those radio-emitting BCGs that were excluded (see Sect. 3.1). Both tables are listed in order of decreasing radio power and contain the following information: Col. $1=$ cluster name, Col. 2 = redshift, Col. 3 = name of the BCG (from NED), Col. $4=$ radio power at $1.4 \mathrm{GHz}$ (see Sect. 3.1 ), Col. $5=$ note on the dynamical state of the cluster (see Sect. 3.2), and Col. $6=$ note on the diffuse cluster scale emission $(\mathrm{RH}=$ radio halo, $\mathrm{MH}=$ mini-halo). The three clusters without obvious BCG are listed in Table 3. Some notes on the special cases are given in the next subsections.

Figure 1 shows the absolute red magnitude for the 44 objects in the sample with optical information available on the SDSS. Those galaxies without information are plotted as crosses at a fixed magnitude. The BCGs with radio emission are circled in black. There seems to be no bias in redshift or cluster type for those BCGs whose magnitude is unavailable on SDSS. Most of the BCGs with available information have an absolute magnitude in the range $-23 \lesssim R \lesssim-24$, with few objects outside this interval. The faintest objects are at the highest redshift in the sample and are notably found in merging clusters. The stellar masses for BCGs in the redshift range considered here are on average a few times $10^{11} M_{\text {Sun }}$ (Lin et al. 2013). 
R. Kale et al.: Radio properties of BCGs in the extended GMRT cluster sample

Table 1. BCG identifications. Radio powers and upper limits for the statistical sample.

\begin{tabular}{|c|c|c|c|c|c|}
\hline Name & $z$ & $\begin{array}{l}\text { BCG ID } \\
\text { 2MASX/SDSS/other }\end{array}$ & $\begin{array}{r}\log P_{1.4 \mathrm{GHz}} \\
\quad \mathrm{W} \mathrm{Hz}-1\end{array}$ & $\begin{array}{l}\text { Dyn. } \\
\text { state }\end{array}$ & $\begin{array}{c}\text { Diff. } \\
\text { emission }\end{array}$ \\
\hline A 1763 & 0.2279 & 1237662306722447498 & 26.09 & $\mathrm{M}^{1}$ & \\
\hline A 2390 & 0.2329 & 1237680297268019748 & 25.54 & $\mathrm{R}^{2}$ & $\mathrm{mH}^{a}$ \\
\hline S 780 & 0.2357 & 2MASXJ14592875-1810453 & 25.21 & $\mathrm{R}^{2}$ & $\mathrm{mH}^{b, c}$ \\
\hline RXC J1115.8+0129 & 0.3499 & 1237654028716802393 & 24.87 & $\mathrm{R}^{2}$ & \\
\hline RX J0439.0+0520 & 0.208 & 2MASXJ04390223+0520443 & 24.85 & $\mathrm{R}^{3}$ & \\
\hline RX J1532.9+3021 & 0.345 & 2MASXJ15012308+4220405 & 24.80 & $\mathrm{R}^{2}$ & $\mathrm{mH}^{d, e}$ \\
\hline A 1835 & 0.252 & 2 MASXJ14010204+0252423 & 24.80 & $\mathrm{R}^{1}$ & $\mathrm{mH}^{f}$ \\
\hline A 1576 & 0.302 & 2MASXJ12365866+6311145 & 24.77 & $\mathrm{R}^{3}$ & \\
\hline A 1300 & 0.3075 & 2MASXJ11315413-1955391 & 24.77 & $\mathrm{M}^{2}$ & $\mathrm{RH}^{g}$ \\
\hline RXC J1504.1-0248 & 0.2153 & 1237655497600467190 & 24.76 & $\mathrm{R}^{2}$ & $\mathrm{mH}^{h}$ \\
\hline RX J2129.6+0005 & 0.235 & 2MASXJ21293995+0005207 & 24.64 & $\mathrm{R}^{4}$ & $\mathrm{mH}^{c}$ \\
\hline A 2667 & 0.2264 & 2MASXJ23513947-2605032 & 24.50 & $\mathrm{R}^{2}$ & \\
\hline $\mathrm{Z} 5247$ & 0.229 & 2MASXJ12342409+0947157 & 24.46 & $\mathrm{M}^{1}$ & \\
\hline A 2146 & 0.234 & 2MASXJ15561395+6620530 & 24.44 & $\mathrm{M}^{3}$ & \\
\hline RX J0027.6+2616 & 0.3649 & 2MASXJ00274579+2616264 & 24.38 & $\mathrm{M}^{3}$ & \\
\hline Z2701 & 0.214 & 2MASXJ09524915+5153053 & 24.32 & $\mathrm{R}^{2}$ & \\
\hline A 1758a & 0.28 & 2 MASXJ13323845+5033351 & 24.27 & $\mathrm{M}^{2}$ & $\mathrm{RH}^{g}$ \\
\hline Z2089 & 0.2347 & 2MASXJ09003684+2053402 & 24.25 & $\mathrm{R}^{2}$ & \\
\hline RX J2228.6+2037 & 0.4177 & 1237680298882433199 & $<24.15$ & $\mathrm{M}^{2}$ & \\
\hline Z2661 & 0.3825 & 1237667733956395341 & $<24.06$ & $\mathrm{M}^{\star}$ & $\mathrm{RH}^{n}{ }^{n}$ \\
\hline A 2261 & 0.224 & 2MASXJ17222717+3207571 & 24.04 & $\mathrm{R}^{2}$ & \\
\hline Z 1953 & 0.373 & 2MASXJ08500730+3604203 & $<24.04$ & $\mathrm{M}^{4}$ & \\
\hline A 2895 & 0.2275 & 2MASXJ01181108-2658122 & 24.02 & $\mathrm{M}^{1}$ & \\
\hline $\mathrm{Z} 7160$ & 0.2578 & 2 MASXJ14571507+2220341 & 23.98 & $\mathrm{R}^{2}$ & $\mathrm{mH}^{i}$ \\
\hline Z3146 & 0.29 & 2MASXJ10233960+0411116 & 23.95 & $\mathrm{R}^{4}$ & $\mathrm{mH}^{d, l}$ \\
\hline A 963 & 0.206 & 2MASXJ10170363+3902500 & 23.92 & $\mathrm{R}^{\star}$ & \\
\hline A 1722 & 0.327 & ABELL1722:[HHP90]1318+7020A & $<23.90$ & $\mathrm{R}^{4}$ & \\
\hline Z348 & 0.254 & 1237666340799643767 & 23.89 & $\mathrm{R}^{3}$ & \\
\hline A 2744 & 0.3066 & ABELL2744:[CN84]001 & $<23.84$ & $\mathrm{M}^{2}$ & $\mathrm{RH}+\mathrm{Re}^{g, m}$ \\
\hline A $2744 \_1$ & 0.3066 & ABELL2744:[CN84]002 & $<23.84$ & $\mathrm{M}^{2}$ & $\mathrm{RH}+\mathrm{Re}^{g, m}$ \\
\hline Z 5699 & 0.3063 & 2MASXJ13055884+2630487 & $<23.84$ & $\mathrm{M}^{3}$ & \\
\hline A 781 & 0.2984 & 2MASXJ09202578+3029380 & $<23.81$ & $\mathrm{M}^{2}$ & $\operatorname{Rel}^{n, o}$ \\
\hline A 2537 & 0.2966 & 2MASXJ23082221-0211315 & $<23.80$ & $\mathrm{R}^{2}$ & \\
\hline A 2813 & 0.2924 & 2 MFGC 00530 & $<23.79$ & $M^{1}$ & \\
\hline Z7215 & 0.2897 & 2 MASXJ15012308+4220405 & $<23.78$ & $\mathrm{M}^{\star}$ & \\
\hline A 2631 & 0.2779 & 2MASXJ23373975+0016165 & $<23.74$ & $\mathrm{M}^{2}$ & \\
\hline RX J0142.0+2131 & 0.28 & {$[\mathrm{BDJ} 2005] 0479$} & 23.72 & $\mathrm{R}^{3}$ & \\
\hline A 1682 & 0.226 & 2MASXJ13064997+4633335 & 23.71 & $\mathrm{M}^{2}$ & \\
\hline RXC J2211.7-0350 & 0.27 & 2MASXJ22114596-0349438 & $<23.71$ & $\mathrm{R}^{\star}$ & \\
\hline Z 5768 & 0.266 & 2MASXJ13114620+2201367 & $<23.70$ & $\mathrm{M}^{3}$ & \\
\hline A 68 & 0.254 & 2MASXJ00370686+0909236 & $<23.65$ & $\mathrm{M}^{1}$ & \\
\hline A 2645 & 0.251 & 2MASXJ23411705-0901110 & $<23.64$ & $\mathrm{M}^{3}$ & \\
\hline A 2485 & 0.2472 & 2MASXJ22483112-1606258 & $<23.62$ & $\mathrm{R}^{3}$ & \\
\hline \multirow[t]{2}{*}{ RXC J1314.4-2515 } & 0.2439 & 2MASXJ13142209-2515456 & $<23.61$ & M & $\mathrm{RH}+2 \operatorname{Rel}^{b, p}$ \\
\hline & & 2MASXJ13143263-2515266 & $<23.61$ & & \\
\hline A 2697 & 0.232 & 2MASXJ00031162-0605305 & $<23.56$ & $\mathrm{R}^{3}$ & \\
\hline RXC J0437.1+0043 & 0.2842 & 2MASXJ04370955+0043533 & 23.55 & $\mathrm{R}^{2}$ & \\
\hline A 3444 & 0.2542 & 2MASXJ10235019-2715232 & 23.55 & $\mathrm{R}^{1}$ & $\mathrm{mH}^{c}$ \\
\hline A 267 & 0.23 & 2MASXJ01524199+0100257 & $<23.55$ & $\mathrm{M}^{2}$ & \\
\hline Z 5247_1 & 0.229 & 2MASXJ12341746+0945577 & $<23.55$ & $\mathrm{M}^{1}$ & \\
\hline A 2111 & 0.229 & 2MASXJ15394049+3425276 & $<23.55$ & $\mathrm{M}^{\star}$ & \\
\hline A 2219 & 0.2281 & 2MASXJ16401981+4642409 & $<23.54$ & $\mathrm{M}^{2}$ & $\mathrm{RH}^{m}$ \\
\hline RXC J1514.9-1523_1 & 0.2226 & 2MASXJ15145772-1523447 & $<23.52$ & $\mathrm{M}^{1}$ & $\mathrm{RH}^{q}$ \\
\hline RXC J1514.9-1523 & 0.2226 & 2MASXJ15150305-1521537 & $<23.52$ & $\mathrm{M}^{1}$ & $\mathrm{RH}^{q}$ \\
\hline RXC J0510.7-0801 & 0.2195 & 2MASXJ05104786-0801449 & $<23.51$ & $\mathrm{M}^{1}$ & \\
\hline A 773 & 0.217 & 2MASXJ09175344+5143379 & $<23.49$ & $\mathrm{M}^{2}$ & $\mathrm{RH}^{r}$ \\
\hline A 1423 & 0.213 & 2MASXJ11571737+3336399 & $<23.48$ & $\mathrm{R}^{2}$ & \\
\hline A 209 & 0.206 & 2MASXJ01315250-1336409 & $<23.44$ & $\mathrm{M}^{2}$ & $\mathrm{RH}^{b}$ \\
\hline A 2163 & 0.203 & ABELL2163:[MCF2008]308 & $<23.43$ & $\mathrm{M}^{2}$ & $\mathrm{RH}^{s}$ \\
\hline
\end{tabular}

Notes. Dynamical state: $\mathrm{M}=$ merger, $\mathrm{R}=$ relaxed; diffuse emission: $\mathrm{mH}=$ mini-halo; $\mathrm{RH}=$ radio halo; Rel = relic. References to dynamical state:

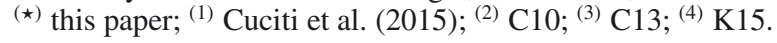

References. References to diffuse emission: ${ }^{(a)}$ Bacchi et al. (2003); ${ }^{(b)} \mathrm{V} 07 ;{ }^{(c)} \mathrm{K} 15 ;{ }^{(d)} \mathrm{K} 13 ;{ }^{(e)}$ Giacintucci et al. $(2014) ;{ }^{(f)}$ Murgia et al. (2009); ${ }^{(g)}$ V13; ${ }^{(h)}$ Giacintucci et al. (2011b); ${ }^{(i)}$ Mazzotta \& Giacintucci (2008); ${ }^{(l)}$ Giacintucci et al. (2014); ${ }^{(m)}$ Orrú et al. (2007); ${ }^{(n)}$ V08; ${ }^{(o)}$ Venturi et al. (2011); ${ }^{(p)}$ Feretti et al. (2005); ${ }^{(q)}$ Giacintucci et al. (2011c); ${ }^{(r)}$ Govoni et al. (2001); ${ }^{(s)}$ Feretti et al. (2001). 
Table 2. BCG identifications. Radio powers for the faint sample.

\begin{tabular}{lllrrc}
\hline \hline Name & $z$ & $\begin{array}{l}\text { BCG ID } \\
\text { 2MASX/SDSS/other }\end{array}$ & $\begin{array}{r}\log P_{1.4 \mathrm{GHz}} \\
\mathrm{W} \mathrm{Hz}^{-1}\end{array}$ & $\begin{array}{c}\text { Dyn. } \\
\text { state }\end{array}$ & $\begin{array}{c}\text { Diff. } \\
\text { emission }\end{array}$ \\
\hline A 2552 & 0.301 & 2MASXJ23113330+0338056 & 23.37 & $\mathrm{R} ?^{1}$ & \\
A 697 & 0.282 & 2MASXJ08425763+3622000 & 23.30 & $\mathrm{M}^{2}$ & $\mathrm{RH}^{a, b}$ \\
A 141 & 0.23 & 2MASXJ01053543-2437476 & 23.21 & $\mathrm{M}^{2}$ & \\
A 773_1 & 0.217 & 2MASXJ09175344+5144009 & 23.21 & $\mathrm{M}^{2}$ & $\mathrm{RH}^{c}$ \\
A611 & 0.288 & 2MASXJ08005684+3603234 & 23.12 & $\mathrm{R}^{2}$ & \\
RX J0439.0+0715 & 0.244 & 2MASXJ04390053+0716038 & 23.12 & $\mathrm{R}^{3}$ & \\
A 2163_1 & 0.203 & 2MASX J16153353-0609167 & 22.99 & $\mathrm{M}^{2}$ & $\mathrm{RH}^{d}$ \\
A 3088 & 0.2537 & 2MASXJ03070207-2839574 & 22.79 & $\mathrm{R}^{2}$ & \\
A 521 & 0.2475 & 2MASXJ04540687-1013247 & 22.66 & $\mathrm{M}^{2}$ & $\mathrm{RH}+\mathrm{Rel}^{e}$ \\
\hline
\end{tabular}

Notes. Dynamical state: $\mathrm{M}=$ merger, $\mathrm{R}=$ relaxed; diffuse emission: $\mathrm{mH}=$ mini-halo; $\mathrm{RH}=$ radio halo; Rel = relic.

References. References to dynamical state: ${ }^{(1)}$ Cuciti et al. (2015); ${ }^{(2)} \mathrm{C} 10$; ${ }^{(3)} \mathrm{C} 13$. References to diffuse emission: ${ }^{(a)}$ V08; ${ }^{(b)}$ Macario et al. (2010); ${ }^{(c)}$ Govoni et al. (2001); ${ }^{(d)}$ Feretti et al. (2001); ${ }^{(e)}$ Brunetti et al. (2008).

Table 3. Clusters without BCG.

\begin{tabular}{lccc}
\hline \hline Name & $z$ & Dynamical state & Note $^{\star}$ \\
\hline RXC J2003.5-2323 & 0.317 & $\mathrm{M}^{1}$ & $\mathrm{GRH}^{a}$ \\
RXC J1212.3-1816 & 0.269 & $\mathrm{M}^{2}$ & - \\
A 520 & 0.203 & $\mathrm{M}^{1}$ & $\mathrm{GRH}^{b}$ \\
\hline
\end{tabular}

Notes. References to dynamical state: ${ }^{(1)} \mathrm{C} 10$; $^{(2)} \mathrm{K} 15 .{ }^{(\star)}$ Note on the diffuse radio emission: $\mathrm{GRH}=$ giant radio halo.

References. ${ }^{(a)}$ Giacintucci et al. (2009); ${ }^{(b)}$ Govoni et al. (2001).

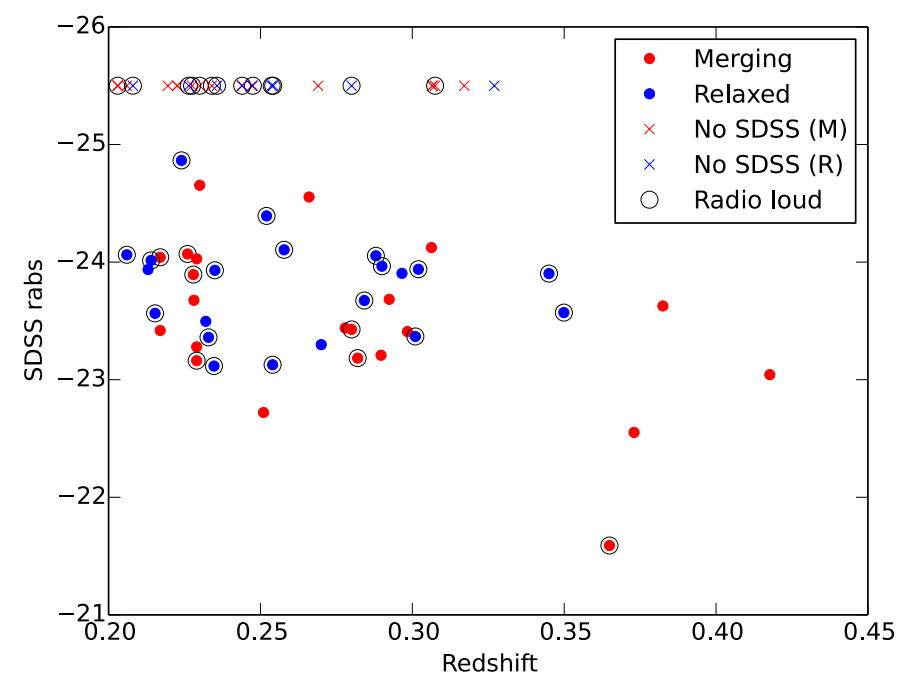

Fig. 1. Distribution of the SDSS absolute red magnitude of the BCGs in the sample. Red and blue dots show the BCGs in merging and relaxed clusters, respectively (see Sect. 3.2). Crosses show the BCGs without optical information, with the same colour code. Black circles show the galaxies with radio emission (see Sect. 3.1 and Tables 1 and 2).

\subsection{Clusters with multiple BCGs}

The cluster sample consists of several dynamically disturbed clusters, some of them with multiple peaks in the X-ray surface brightness images. The identification of the BCG in these clusters was made under the hypothesis that more than one BCG may be present, possibly associated with merging sub-clusters.
We considered only those BCGs falling within the X-ray emission of the host cluster. Below we report some information.

- A 773 is a merging cluster with a radio halo (Govoni et al. 2001). Two BCGs are located close to the single X-ray peak. One has a compact radio source, detected after re-analyis of archival VLA observations at $1.4 \mathrm{GHz}$ (Tables 1 and 2).

- A 2163 has two BCGs (Maurorgodato et al. 2008). One of them is radio quiet, while the radio power of the second one is below the threshold considered for our statistical studies (Sect. 3.1, Tables 1 and 2).

- A 2744 has two BGCs, located at the peak of both the X-ray emission and the radio halo (V13, Giacintucci et al., in prep.). Both galaxies are radio quiet (Table 1).

- RXC J1314.4-2515 is a known merging system (Mazzotta et al. 2011) with two radio relics and a halo (V07, Feretti et al. 2005). Two BCGs were identified by Valtchanov et al. (2002). They lie on each side of the single X-ray peak and are both radio quiet (Table 1).

- RXC J1514.9-1523 has two BGCs, both are radio quiet.

- Z 5247 has a double-peaked X-ray morphology with a BCG at each peak. One of the two BCGs has detected radio emission (Table 1).

Only three out of ten of the BCGs in these multiple-merger clusters show radio emission at some level.

\subsection{Clusters without BCGs}

In the optical field of A 520, RXC J2003.5-2323, and RXC J1212.3-1816 there is no dominant galaxy that can be considered a BCG. These three are all merging clusters, two of them with a radio halo (see Table 3 ).

Three BCGs have been reported in the literature for A 520 (Mahdavi et al. 2007), but they are all very distant from the centre of the X-ray emission, and their absolute optical magnitude is some magnitudes fainter than typical for this class of objects, hence we regard A 520 as a non-BCG cluster.

\subsection{Other special cases}

The galaxy cluster A 141 shows a complex X-ray substructure with a prominent secondary peak south of the main double condensation. Based on the image inspection and on the literature information, we considered only the BCG listed in 
Hoffer et al. (2012) as valid. The radio power of this galaxy is below the threshold considered for our statistical investigations (see Sect. 3.1) and is reported in Table 2.

The central region of A 2813 is quite complex, with three candidate galaxies within the brightest region of X-ray emission. According to the information available from NED (and by visual inspection of DSS-2), the galaxy coincident with the peak of the $\mathrm{X}$-ray emission is the faintest. The brightest galaxy in this region is 2 MFGC 00530, and we consider it as the cluster BCG. It is radio quiet (see Table 1).

\section{Radio and X-ray data}

\subsection{Radio data}

The EGRHS is the starting point of our BCG sample. The radio information we used to derive the radio luminosity function, however, was not taken from the $610 \mathrm{MHz}$ GMRT observations (V07, V08, K13, and K15). To ensure a sensitivity as uniform as possible over the whole sample and enable a direct comparison with works from other authors, which were mainly performed at $1.4 \mathrm{GHz}$, we cross-checked our sample with the Northern VLA Sky Survey (NVSS) and extracted the $1.4 \mathrm{GHz}$ flux density of each source directly from those images. To overcome a few cases of blending on NVSS, we used the images on the VLA FIRST Survey where possible (angular resolution of $5^{\prime \prime}$ ). Finally, for BCGs in mini-halo clusters (see Table 1) we used the $1.4 \mathrm{GHz}$ flux density values published in Giacintucci et al. (2014), which were accurately estimated to avoid contamination from the diffuse emission of the mini-halo ${ }^{1}$.

The resolution of FIRST images is comparable to our $610 \mathrm{MHz}$ GMRT images $\left(\sim 5^{\prime \prime}\right)$, but they are not available for a number of clusters, and the angular resolution of the NVSS $\left(45^{\prime \prime} \times 45^{\prime \prime}\right)$ is inadequate to isolate the flux density of the BCG from that of other nearby sources (RXC J0439.0+0520, RXC J0027.6+2616, Z 348, RXC J0142.0+2131 and A 1682) or from a diffuse radio halo (A 1300 and A 1758a). In these cases we used our high-resolution images at $610 \mathrm{MHz}$ (V07, V08, $\mathrm{K} 13$, and K15) and at $325 \mathrm{MHz}$ (Venturi et al. 2013) and derived the flux density at $1.4 \mathrm{GHz}$ assuming a spectral index of 0.8 , which is a reasonable average value for this type of sources (Klein et al. 1995).

Because the EGRHS has a higher sensitivity than NVSS and FIRST, six BCGs detected at $610 \mathrm{MHz}$ do not have a counterpart either in NVSS or FIRST. These are A 141 and A 3088 (V07), A 521 (Giacintucci et al. 2006), A 697 (V08), A 2552 (K15), and RXJ 0439.0+0715 (K13). Moreover, radio emission below the sensitivity limit of FIRST and NVSS was detected by Giacintucci et al. (in prep.) for A 773_1 and A 2163_1 after re-analysing archival $1.4 \mathrm{GHz}$ VLA data. Finally, A 611 has a strong detection at $610 \mathrm{MHz}$ (the radio source associated with the BCG has $S_{610 \mathrm{MHz}}=59 \pm 3 \mathrm{mJy}$ ), but nothing is visible on NVSS. Inspection of the FIRST image shows a very weak source (at $\sim 4 \sigma$ ) that would have remained unnoticed without careful comparison with the $610 \mathrm{MHz}$ image. For this source we report in Table 2 the radio power derived from the $1.4 \mathrm{GHz}$ flux density from FIRST. The nine radio sources listed in Table 2 were removed from our statistical analysis.

For BCGs without radio emission, we considered a conservative radio power upper limit derived from NVSS, whose average

\footnotetext{
1 For the BCG in S 780, flux density measurements from GMRT proprietary data and re-analysis of VLA archival data suggest that the source is variable and that the core of the radio emission has an inverted spectrum.
}

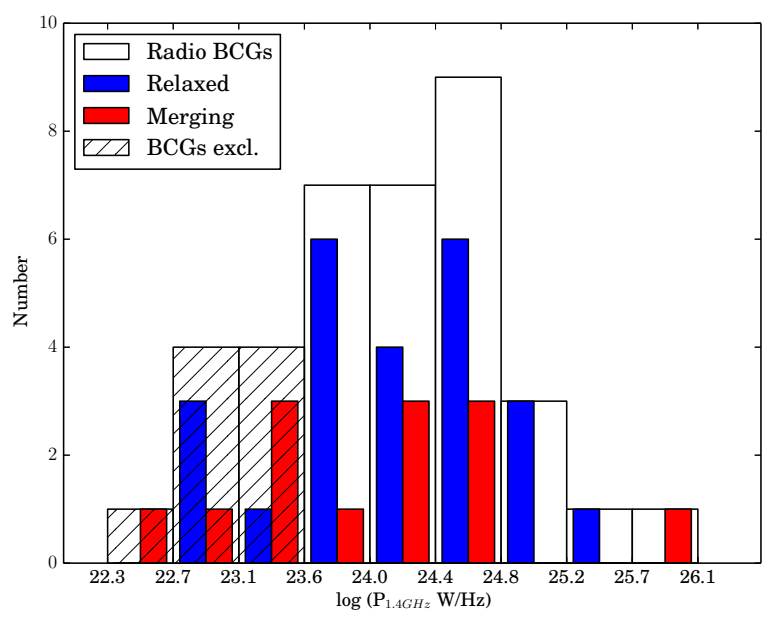

Fig. 2. Histogram of the radio power of the BCGs in the sample. The shaded intervals refer to the BCGs that were removed from the statistical analysis (Sect. 3.1).

noise level is $1 \sigma=0.45 \mathrm{mJy} \mathrm{b}^{-1}$, that is, $S_{1.4 \mathrm{GHz}} \leq 2.25 \mathrm{mJy}$ (namely $5 \sigma$, see Table 1 ).

The final sample we used for the statistical studies (see Sects. 4 and 5) includes a total of 59 BCGs, hosted in 55 clusters.

The histogram in Fig. 2 shows the radio power distribution of all BCGs with radio emission, including the nine faintest objects (see Table 2) that we did not consider in the statistical analysis performed in Sect. 4. The distribution peaks around $\log P_{1.4 \mathrm{GHz}}\left(\mathrm{W} \mathrm{Hz}^{-1}\right) \sim 24.5$, which is the typical transition power between FRI and FRII radio galaxies (Fanaroff \& Riley 1974), as commonly found at cluster centres. Except for the case of the BCG in A 1763 (the only object in the bin of highest radio power), the BCGs in relaxed clusters are the most abundant in most bins of radio power.

As a final remark, we note that all the radio BCGs in our sample show very little extended structure at $610 \mathrm{MHz}$ and $1.4 \mathrm{GHz}$. The only exception is the wide-angle tail (WAT) at the centre of A 1763, whose $1.4 \mathrm{GHz}$ contours from FIRST are shown in Fig. 3 overlaid on the optical frame and on the Chandra X-ray emission. This is also the most powerful radio source in the sample (see Table 1). WAT radio galaxies are found only in association with brightest cluster galaxies (Feretti \& Venturi 2002, for a review), and their bent morphology is considered as the signature of bulk motions in the ICM (Burns 1998).

\subsection{X-ray data and cluster morphological analysis}

To assess the cluster dynamical status, we used the morphological parameters, namely the power ratio $P_{3} / P_{0}$, the centroid shift $w_{500}$, and the concentration parameter $c_{100}$, derived in $\mathrm{C} 10$ and Cassano et al. (2013, hereafter C13) from proprietary and archival Chandra data.

We briefly recall here that the power ratio is a multipole decomposition of the two-dimensional projected mass within a given aperture, and it provides a measure of the substructure (e.g. Buote \& Tsai 1995). The centroid shift $w$ is defined as the standard deviation of the projected separation between the peak and the centroid of the cluster X-ray brightness distribution (e.g. Poole et al. 2006). In particular, $w_{500}$ is estimated over an aperture of $500 \mathrm{kpc}$. The concentration parameter $c_{100}$ is defined as the ratio of the peak (within $100 \mathrm{kpc}$ ) over the ambient (within $500 \mathrm{kpc}$ ) X-ray surface brightness (Santos et al. 2008).

For five clusters not included in these earlier works (marked with $\star$ in Table 1) we derived the morphological indicators 

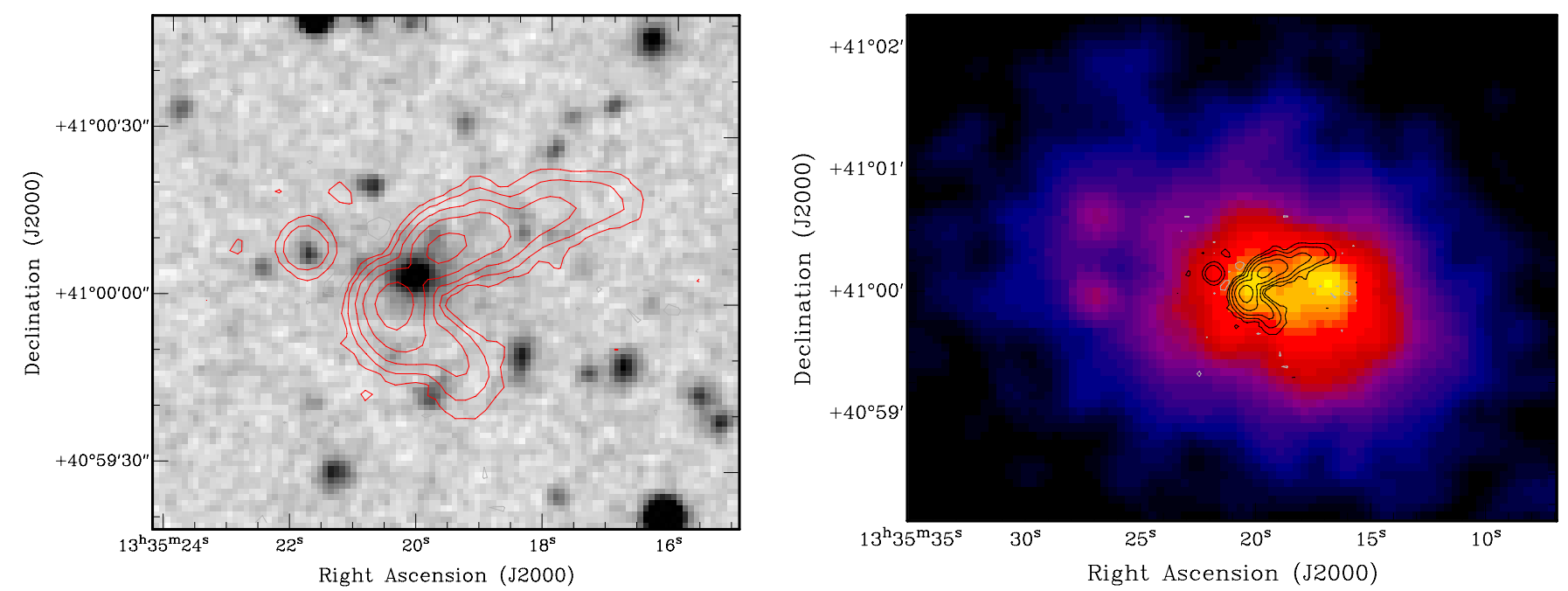

Fig. 3. $1.4 \mathrm{GHz}$ contours from FIRST of the wide-angle tail in A 1763 overlaid on the red optical frame of the ESO Digitized Sky Survey DSS2 (left panel) and on the Chandra image (right panel). Radio contours are $\pm 0.4,1.6,6.4,25.6$, and $102.4 \mathrm{mJy} / \mathrm{b}$ in both panels (negative contours are shown in grey).

following C10 (see Sect. 3 of C10 for details). The results of this analysis are reported in Tables $1-3$, where the clusters are classified as merger $(\mathrm{M})$ or relaxed $(\mathrm{R})$, according to their position in the morphological diagrams.

We further complemented our analysis with literature information, by visual inspection of the available X-ray images, and considering the diffuse extended emission in the form of radio halos or mini-halos, whose connection with merging and relaxed clusters, respectively, is an established result (Brunetti \& Jones 2014).

All 65 clusters in the EGRHS have a classification of their dynamical status: 35 are merging $(\mathrm{M})$ and 30 are relaxed $(\mathrm{R})$, which is $54 \%$ and $46 \%$, respectively (see Tables $1-3$ ). We note that the morphological parameters used to derive the cluster dynamics are not sensitive to mergers aligned close to the line of sight, but it is reasonable to assume that they are only a negligible fraction of the whole esample. The redshift distribution of the two subsamples is quite similar: the median value of $z$ is 0.251 and 0.253 for the merging and relaxed clusters, respectively.

\section{Radio loudness, cluster dynamics, and radio luminosity function}

\subsection{Radio-loudness fraction and cluster dynamics}

Starting from the initial sample of 68 BCGs and after inspecting the radio information available (Sect. 3.1), our final sample contains 59 objects for our statistical analysis. Of these 59 BCGs, 28 are radio loud and 31 are radio quiet (47\% and 53\% in each class, see Table 1).

We divided the 55 clusters hosting the 59 BCGs (see Sect. 2.1 for the clusters with multiple BCGs) into merging and relaxed, following Sect. 3.2 (see Col. 5 in Table 1), to estimate the fraction of radio-loud BCGs in each class: we have 29 merging and 26 relaxed clusters $(53 \%$ and $47 \%$ of the total, respectively). Our results are summarised in Table 4 . Radio-loud BCGs are considerably more common in relaxed clusters $(71 \%$ against $29 \%$ in merging clusters). Conversely, radio-quiet BCGs are much more common in merging systems ( $81 \%$ to be compared to the $19 \%$ in relaxed clusters).

We tested the significance of this result by running Monte Carlo (MC) calculations. In particular, we randomly assigned
Table 4. Radio-loudness fraction in merging and relaxed clusters.

\begin{tabular}{lrrcc}
\hline \hline \# BCGs & Merging & Relaxed & \% Merging & \% Relaxed \\
\hline Radio loud & 8 & 20 & $29 \%$ & $71 \%$ \\
Radio quiet & 25 & 6 & $81 \%$ & $19 \%$ \\
\hline
\end{tabular}

the 28 radio-loud BCGs among the 55 clusters in the sample and counted the number of objects that fall in relaxed clusters in our MC trials. In the left panel of Fig. 4 we report the distribution of the number of radio-loud BCGs in relaxed clusters obtained after $10^{5} \mathrm{MC}$ trials. The distribution can be fitted with a Gaussian function, with a central value of 13.6 and standard deviation of 1.875. This means that the observed value of $20 \mathrm{BCG}$ in relaxed clusters (red point in Fig. 4, left panel) is at $3.4 \sigma$ from the value expected assuming that the distribution of the radio loud BCGs is independent of the cluster dynamical status. This shows that the probability that our result is a chance detection is $\leq 3.4 \times 10^{-4}$. A similar result can be obtained from considering radio-quiet BCGs in merging and relaxed clusters.

The distribution of the BCGs in the different environments is given in the right panel of Fig. 4, which shows the clusters in the $w_{500}-c_{100}$ space, selected to describe the dynamical state. The grey dotted lines are traced as reference values to statistically pinpoint the regions of merging (bottom right portion) and relaxed (upper left portion) clusters (see C10 for details). The black points show the radio loud BCGs, and again we note that the bulk of them $(71 \%)$ are found in relaxed systems.

\subsection{Radio power of BCGs and cluster dynamics}

The radio power of the BCGs in the full sample of 68 objects spans more than three orders of magnitude, from $\log P_{1.4 \mathrm{GHz}} \quad\left(\mathrm{W} \mathrm{Hz}^{-1}\right)=22.66 \quad(\mathrm{~A} 521)$ to $\log P_{1.4 \mathrm{GHz}}\left(\mathrm{W} \mathrm{Hz}^{-1}\right)=26.09$ (A 1763) (see Tables 1 and 2). Even restricting our considerations to the 59 BCGs used in the statistical sample, the range of values is quite broad (2.5 orders of magnitude).

We checked for a possible connection between the radio power of the BCGs, the X-ray luminosity $\left(L_{X}\right)$, and the 

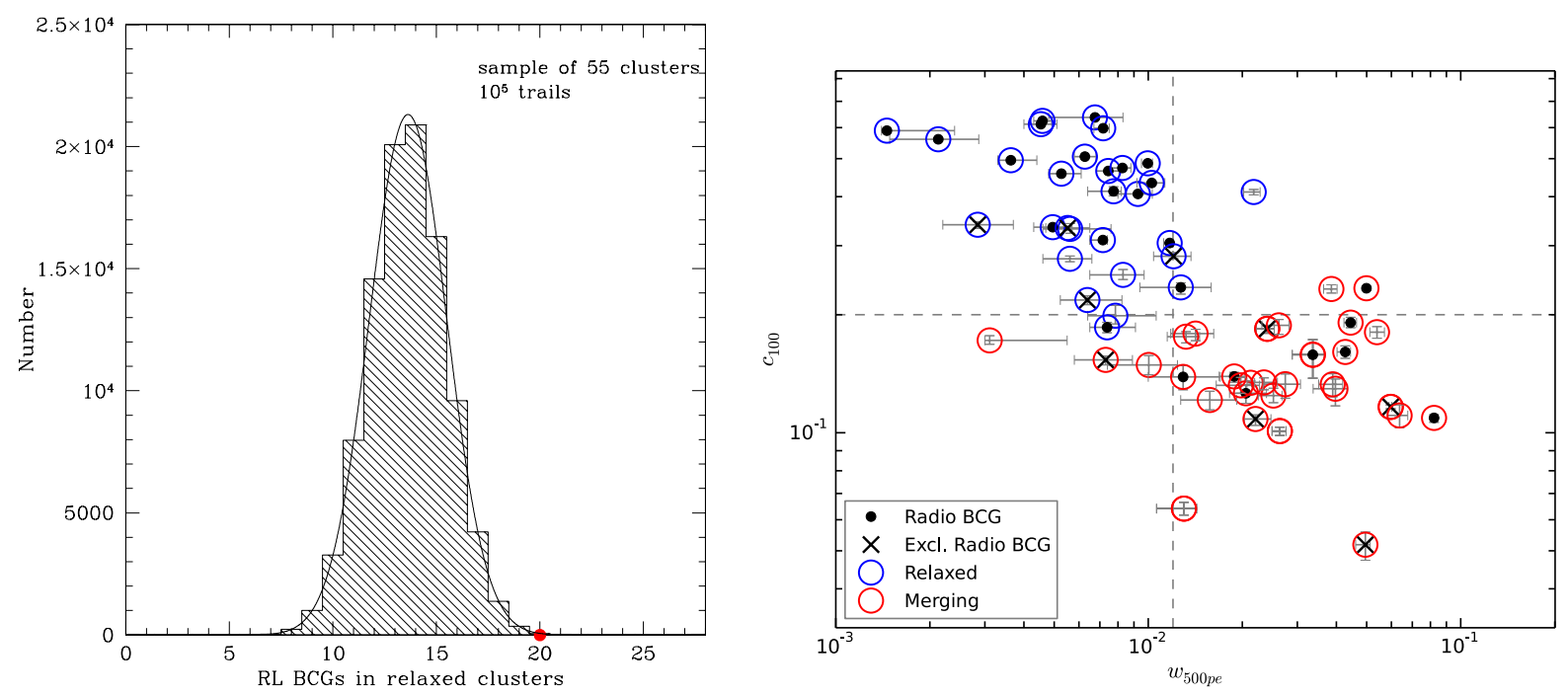

Fig. 4. Left panel: result of the Monte Carlo calculations. The histogram shows the distribution of the radio-loud BCGs in relaxed clusters after $10^{5}$ trials. The red dots shows the $3.4 \sigma$ location of our observed result compared to the random distribution. Right panel: distribution of the $\mathrm{BCG}$ in the $w_{500}-c_{100}$ space. Relaxed clusters are shown as blue circles, and are all located in the upper left quadrant; merging clusters are shown as red cicles and occupy the lower right portion. Filled black circles are the radio-loud BCGs, the black crosses show the BCGs with radio emission that have been removed from the analysis (Sect. 3.1). The threshold values to classify clusters as mergers are the same as in C10, i.e., $P_{3} / P_{0}>1.2 \times 10^{-7}, w_{500}>0.012$ and $c_{100}<0.20$.
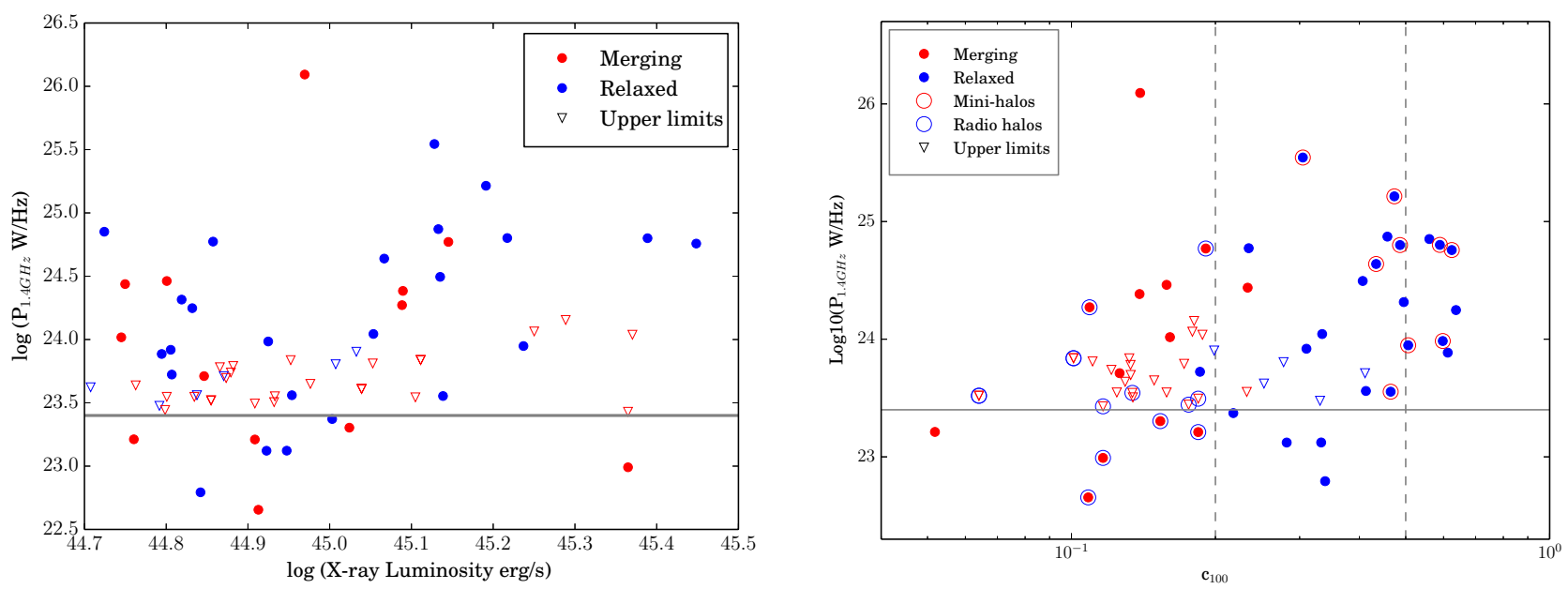

Fig. 5. Left panel: distribution of the $\log L_{\mathrm{X}}-\log P_{1.4 \mathrm{GHz}}$ for the sample, where $\log L_{\mathrm{X}}$ is the X-ray luminosity of the host cluster. Undetected BCGs are shown as triangles (blue for relaxed, red for merging clusters). The objects below the grey line are those undetected in NVSS that were removed from the statistical study. Right panel: distribution of the radio power of the BCGs in the sample as a function of the concentration parameter of the hosting cluster $\left(c_{100}\right)$. Merging clusters are shown as red dots, relaxed clusters are blue circles. Upper limits in each class are shown as triangles, with the same colour code. The information on the presence of mini-halos in relaxed systems and radio halos in merging clusters is also shown. The grey horizontal line is the same as in the left panel. Dashed vertical lines are drawn at $c_{100}=0.2$ and 0.5 (see Sect. 5.1).

dynamical state of the host cluster. The left panel of Fig. 5 shows the distribution of the radio power of the BCGs in different environments versus $L_{\mathrm{X}}$. Upper limits are also plotted. The figure is suggestive of a few considerations. Even though no significant trend is visible, upper limits are much more abundant in less luminous $\left(\log L_{\mathrm{X}}\left(\mathrm{erg} \mathrm{s}^{-1}\right) \leq 45.1\right)$ merging clusters, whereas for $\log L_{X}\left(\mathrm{erg} \mathrm{s}^{-1}\right)>45.1$ the fraction of radio BCGs is much higher, and nine out of ten are found in relaxed clusters.

If we consider the BCGs in relaxed clusters, only a weak trend is present between the radio power and the core X-ray luminosity (within $0.15 R_{500}{ }^{2}$, see C13) of the host cluster: a Spearman test on a possible correlation between these

\footnotetext{
${ }^{2} R_{500}$ is the radius corresponding to a total density contrast $500 \rho_{\mathrm{c}}(z)$, where $\rho_{\mathrm{c}}(z)$ is the critical density.
}

two variables provides $\rho=0.375$ and a probability of null hypothesis of $16.8 \%$. A correlation might be present for $\log L_{X}\left(\mathrm{erg} \mathrm{s}^{-1}\right) \gtrsim 45$, but the small number of points above this value does not allow drawing any conclusion.

Finally, we checked for a possible dependence of the BCG radio power on the cluster dynamics. We used the concentration parameter $c_{100}$ as proxy for the cluster dynamical state (high values of $c_{100}$ indicate peaked X-ray brightness distributions, typical of relaxed clusters) and plotted the radio power and upper limits vs. $c_{100}$. Our results are shown in the right panel of Fig. 5. As additional information, we highlight the relaxed clusters that host a mini-halo and the merging clusters that host a giant radio halo. By definition, all relaxed clusters (blue dots) have $c_{100} \geq 0.2$. 
The radio power range is populated fairly uniformly for both classes. No obvious trend is visible for the radio-loud BCGs in merging clusters, which span the whole range of radio power. On the other hand, the right panel of Fig. 5 is suggestive of a positive trend for the BCGs in relaxed systems, which show increasing radio power with increasing value of $c_{100}$. A Spearman test on the possible correlation between $\log P_{1.4 \mathrm{GHz}}$ and $c_{100}$ for the radio BCGs in relaxed clusters provides a Spearman rankorder coefficient $\rho=0.53$ (suggesting a positive correlation) and a probability of null hypothesis of $1 \%$, which is thus rejected. The test was performed on all relaxed clusters with $c_{100}>0.2$. The inclusion of the two most deviating points (i.e., A 1576 and A 2390) does not change the result.

\subsection{Fractional radio luminosity function}

The fractional radio luminosity function (RLF) is a powerful tool for investigating the statistical properties of a population of objects. It provides the probability of a galaxy to be radio loud with a radio power higher than a given value of $P$.

To minimise the problems raised by the sensitivity limits of different arrays and ensure uniform sensitivity, we based our analysis on NVSS (see Sect. 3.1). However, the redshift of the BCGs in our sample spans over the range $z=0.2-0.4$, and the radio power upper limit for the undetected sources is a function of redshift, as is clear from Table 1. To account for the upper limits in the fractional RLF, different methods have been proposed. Of these, we adopted the method developed by Fanti (see appendix in Hummel 1981), which shows the smallest statistical fluctuations in Monte Carlo experiments. The cumulative fractional radio luminosity function $F\left(\geq P_{k}\right)$ is described as follows:

$F\left(\geq P_{k}\right)=\sum_{j=1}^{k} f\left(P_{j}\right)$

with

$f\left(P_{k}\right)=\frac{1-\sum_{j=1}^{k-1} f\left(P_{j}\right)}{n_{\mathrm{u}}\left(P_{i}<P_{k}\right)+n_{\mathrm{d}}\left(P \leq P_{k}\right)}$.

Here $f\left(P_{j}\right)$ is the fraction of detections in the $j$ th radio power interval, $n_{\mathrm{u}}\left(P_{i}<P_{k}\right)$ is the number of upper limits (undetections) for $P_{i}<P_{k}$, and $n_{\mathrm{d}}\left(P \leq P_{k}\right)$ is the number of detections for $P \leq P_{k}$. Given a sample with $\mathrm{N}$ objects, $n_{\mathrm{u}}$ objects are undetected as a result of sensitivity limits, and $n_{\mathrm{d}}$ are detected, and $n_{\mathrm{u}}+n_{\mathrm{d}}=N$.

We computed the fractional RLF using radio power intervals with width $\Delta \log P_{1.4 \mathrm{GHz}}=0.4$. We then summed the detections in each bin to obtain the RLF in the cumulative form. Our results are shown in Fig. 6, where the RLF is reported for the merging (red) and relaxed clusters (blue).

The fractional RLF for our two subsamples is different. BCGs in relaxed clusters show a significantly higher probability to be radio loud than those in merging systems. At high radio powers the statistics is poor (as is clear from Table 1), while the faint end of the radio luminosity function suffers from incompleteness. Nevertheless, the differences are clear in the most populated bins of radio detections. In particular, the probability that a BCG in a relaxed cluster is radio loud with radio power $\log P_{1.4 \mathrm{GHz}}\left(\mathrm{W} \mathrm{Hz}{ }^{-1}\right) \gtrsim 23.5$ is $\sim 90 \%$, to be compared to $\sim 30 \%$ for the BCGs in merging clusters.

To quantify this result, we applied a Kolmogorov-Smirnov (KS) test to the fractional RLF in merging and relaxed clusters. The null hypothesis in this test is that both samples are drawn

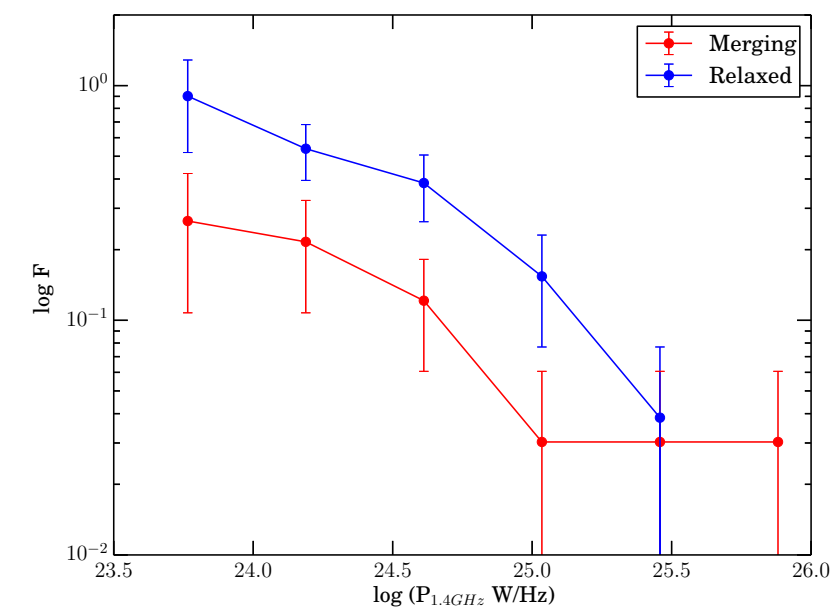

Fig. 6. Cumulative radio luminosity function for the BCGs in merging (red) and relaxed clusters (blue). $F$ is defined in Sect. 4.3. The points are plotted in the middle value of each bin.

from the same distribution. We obtained $D=0.67$ (distribution parameter) and $p=7.6 \%$ (probability function), hence we reject the null hypothesis. The statistical significance of this result is small, but it is strengthened by the results found in the previous sections.

\section{Discussion}

We address the question of a possible connection between the nuclear radio emission of BCGs and the dynamical state of the host cluster. The sample selected to this aim consists of 68 galaxies in the clusters of the Extended GMRT Radio Halo Survey $\left(L_{\mathrm{X}}\right.$ $(0.1-2.4 \mathrm{keV})>5 \times 10^{44} \mathrm{erg} \mathrm{s}^{-1}, 0.2 \leq z \leq 0.4$ and $\left.\delta>-31^{\circ}\right)$.

Previous studies focussed on samples of BCGs in nearby clusters and groups $(z<0.2)$ and were mainly performed in the framework of the AGN/ICM feedback mechanisms, hence they mainly concentrated on the connection between the radio loudness of the BCGs and the presence of a cool core (Mittal et al. 2009; Sun et al. 2009; Dunn \& Fabian 2008). They provided clear indications of a positive correlation between the strength of the cluster cool core and a radio loud BCG, and its power.

The statistical analysis presented here differs from the earlier investigations and complements their results. Our BCG sample covers a new redshift space and extends the findings of previous studies up to $z=0.4$. Our starting sample of galaxy clusters has no a priori bias towards the cluster dynamical state, the clusters being almost evenly distributed between mergers and relaxed systems (52\% and 48\%, respectively). Our analysis is not restricted to the most powerful and extreme BCGs in radio wavelengths, but also includes relatively faint objects, down to $\log P_{1.4 \mathrm{GHz}}\left(\mathrm{W} \mathrm{Hz}^{-1}\right) \sim 23.5$. Finally, our statistical analysis accounts for the sensitivity limits of the radio data, with the inclusions of the upper limits for the non-detections, and it is hence complete within the volume covered by the sample.

\subsection{Statistical properties of the BCGs in the EGRHS cluster sample and the cluster dynamics}

The radio power range of our sample is typical of low- and intermediate-power radio-loud AGN (23 $\log P_{1.4 \mathrm{GHz}} \mathrm{W} \mathrm{Hz}^{-1} \lesssim 26$, see Owen \& Laing 1989 for reference values), and our analysis showed that the nuclear radio properties of BCGs strongly depend on the central properties of the host cluster. 
Roughly $50 \%$ of the BCGs in our sample are radio loud, consistent with earlier findings (Best et al. 2007). However, when we separated the BCGs according to the dynamical state of the cluster, we found that radio-loud BCGs are much more abundant in relaxed systems, that is, $71 \%$ vs. $29 \%$. Monte Carlo simulations showed that the probability of a chance result can be rejected (Sect. 4.1). At the same time, radio-quiet objects were mainly found in merging clusters, that is, $81 \%$ vs. $19 \%$. Motivated by this result, we further investigated the possible dependence of the radio power of the BCGs with the cluster dynamics.

Mittal et al. (2009) reported that at fixed X-ray luminosity the fraction of radio-loud BCGs as well as their radio power increase with increasing cool-core strength. Our analysis based on the X-ray images for the clusters in our sample neither separated cool-core and non-cool-core clusters, nor did it allow measuring the strength of cool cores; however, the parameter $c_{100}$ was introduced to search for cool-core clusters, and its value is a measure of the cool-core strength, which increases with increasing $c_{100}$ (Santos et al. 2008, C13). On this basis, we compared our results and those in Mittal et al. (2009) and tentatively separated all clusters into three different intervals of $c_{100}$, following their classification (see dotted lines in the right panel of Fig. 5): clusters with $c_{100}<0.2$ are mergers, that is, most likely non-cool-core clusters (NCC), those with $0.2<c_{100}<0.5$ are weak cool-cores (WCC), and those with $c_{100}>0.5$ probably have strong cool-cores (SCC). Allowing for the uncertainty due to the poorer statistics in each $c_{100}$ interval, our data are consistent with the result reported in Mittal et al. (2009). In particular, the fraction of radio BCGs is considerably lower in clusters with $c_{100}<0.2(\mathrm{NCC})$ than in WCC $+\mathrm{SCC}$ clusters $\left(c_{100}>0.2\right)$ : it rises from $\sim 33 \%$ to $\sim 85 \%$. If we restrict our considerations to the WCC and SCC clusters, the fraction further increases from $\sim 84 \%$ for WCC to $100 \%$ for SCC, as is clear in Fig. 5 (right).

Finally, we found a positive correlation between the concentration parameter and the radio power of the BCG in relaxed clusters. After removing A 1576 and A 2390 (see Sect. 4.1), we fitted the law

$\log P_{10}(1.4 \mathrm{GHz})=\mathrm{a} \log _{10} c_{100}+b$

and found $a=2.55 \pm 0.33$ and $b=25.05 \pm 0.83$. For comparison, Mittal et al. (2009) found $P_{1.4 \mathrm{GHz}} \propto t_{\text {cool }}^{-3.16 \pm 0.38}$, on a larger sample of objects. Considering that $t_{\text {cool }} \propto c_{100}^{-1}$ (e.g. Santos et al. 2008), our results are in line with those obtained by Mittal et al. (2009).

The close connection between the fraction of radio loud BCGs, their power, and the cluster dynamics is further strengthened by the fractional radio luminosity function (RLF), which shows that BCGs in relaxed systems have a considerably higher probability to be radio loud than those in merging clusters: the probability to find a radio loud $\mathrm{BCG}$ with $\log P_{1.4 \mathrm{GHz}} \mathrm{W} \mathrm{Hz}{ }^{-1} \gtrsim$ 23.5 is almost $90 \%$ for relaxed clusters, while it falls to $\sim 20-30 \%$ in merging clusters.

\subsection{Optical properties of the BCGs in the EGRH sample}

To check for possible trends between the dynamical state of the host clusters, the radio emission of the BCGs and the properties of the gaseous environment feeding the AGN, we investigated the occurrence of optical emission lines in the spectra of the BGCs in our sample.

Unfortunately, only 28/68 BCGs in the full sample have spectra on the SDSS, therefore no strong conclusions can be drawn. Nevertheless, the results are interesting. Nineteen of these 28 BCGs show radio emission ( 5 out of 19 in merging and 14 out of 19 in relaxed clusters), and 9 have upper limits ( 7 out of 9 in merging and 2 out of 9 in relaxed clusters). None of the galaxies with radio upper limit shows emission lines in the optical spectrum, while 11 out of 19 radio loud do, and they are all found in relaxed clusters.

The optical spectrum of the BCG is available only for seven of the mini-halo clusters in our sample, and emission lines are present in all cases. Those are A 1835, A 2390, RXC J1504.10248，RXC J1532.9+3021，RXC J2129.6+0005，Z 3146， and Z7160. The remaining four are RXC J1115.8+0129, Z 2089, Z2701, and Z348.

It is interesting to note that the radio power of these emission line galaxies in our sample is typical of low- to intermediate-power radio galaxies, which usually lack emission lines (Balmaverde et al. 2008; Heckman \& Best 2014, and references therein). Moreover, most of the radio-emitting BCGs in the sample are unresolved. The only exceptions are the small tail in Z 5247 and the large WAT in A 1763 (see Fig. 3).

\subsection{BCGs, accretion, and the radio properties of the host clusters}

The dynamical state of galaxy clusters and their overall formation through a series of mergers are phenomena involving scales much larger $(\mathrm{Mpc})$ than the inner regions at play in the radioloud activity of AGN, whose typical sizes are of the order of the sub-kpc. However, the striking occurrence of radio-loud BCGs in relaxed clusters and the positive trend between radio power and cool-core strength suggest that the two phenomena are related, at least to some extent.

The radio emission in massive early-type galaxies is broadly classified on the basis of their accretion rate, with jet-mode radio galaxies accreting inefficiently $\left(L / L_{\text {Edd }} \lesssim 0.01\right)$ and radiativemode sources accreting at high rates, that is, $L / L_{\text {Edd }} \gtrsim 0.1$ (see Heckman \& Best 2014 for a recent review and nomenclature). The two classes of radio-loud AGN differ in the optical properties, the former being low-excitation (LERG), the latter being high-excitation galaxies (HERG). Typically, radio galaxies with moderate radio power $\left(P_{1.4 \mathrm{GHz}} \lesssim 10^{25} \mathrm{~W} \mathrm{~Hz}^{-1}\right)$ belong to the first class (e.g. Balmaverde et al. 2008), while more powerful radio galaxies $\left(P_{1.4 \mathrm{GHz}} \gtrsim 10^{25} \mathrm{~W} \mathrm{~Hz}^{-1}\right)$ usually show spectral features typical of the HERG class. It has recently been proposed (Hardcastle et al. 2007) that a main difference between LERG and HERG resides in the source of accreting gas: LERG may accrete hot gas from the intergalactic medium (hot-mode), while HERG may be fed by infalling cold gas (cold-mode). It has been further pointed out that the radio luminosity function for hotmode radio galaxies would depend both on the black hole mass function and on the distribution properties of the central hot gas, while that of the cold-mode radio galaxies would not be affected by the black hole mass.

By definition, cool-core clusters are characterised by relatively cold gas with high mass-deposition rates at their centres (see Hudson et al. 2010 for a review). This builds up a reservoir of cold gas for the BCGs at their centres, which may feed the AGN and provide fuel to the radio emission. Even if only in a limited fraction of our sample, the available spectral information does support this, suggesting that at least part of the radio-loud BCGs in cool-core clusters in our sample are supplied by cold gas at the cluster centre. An impressive example is the BCG in RXC J1504.1-0248 (Ogrean et al. 2010), which is one of the cool-core clusters with a mini-halo. At the same time, the observations show that cool cores are often disrupted during cluster 
mergers, and this cold gas may no longer be available to the BCGs in unrelaxed systems. Indeed, none of the emission line BCGs in our sub-sample (see previous section) is in a merging cluster.

It is tempting to suggest that the fraction of radio galaxies and the fractional radio luminosity function for the BCGs in the relaxed clusters of our sample is the result of two populations of radio galaxies, one accreting gas from the hot corona, and the other accreting cold gas in the cluster core region. The latter would not be found in merging clusters, as the contribution of the intracluster cold gas would be lacking.

\section{Summary and conclusions}

We studied the possible connection between the radio properties of brightest cluster galaxies and the dynamical state of the host cluster using a sample of BCGs selected from the Extended GMRT Radio Halo Sample (EGRHS). The BCGs in our sample are located in the redshift interval $0.2 \leq z \leq 0.4$. All clusters have available quantitative information on their dynamical state from Chandra X-ray data. We can summarise our results as follows.

- Most of the BCGs in our sample have optical red magnitudes in the range $-24 \lesssim R \lesssim-23$, that is, the stellar mass range of the galaxies (a few times $10^{11} M_{\text {Sun }}$ ) is narrow. Because the fraction of radio galaxies and the radio luminosity function depend on the stellar mass (e.g. Auriemma et al. 1977; Ledlow \& Owen 1996; Bardelli et al. 2010), the optical properties of our sample ensure that our results are not strongly affected by this.

- The full radio sample includes 68 BCGs, whose radio power spans a wide range, from $\log P_{1.4 \mathrm{GHz}}\left(\mathrm{W} \mathrm{Hz}^{-1}\right)=22.8$ to 26.1. Most of the radio galaxies are unresolved at the resolution of a few arcsec (GMRT at $610 \mathrm{MHz}$ ).

- High-quality X-ray imaging is available for all the 65 clusters in the EGRHS. Our quantitative morphological analysis shows that merging and relaxed clusters are fairly equally represented with $54 \%$ and $46 \%$, respectively.

- Fourty-seven percent of the sample of 59 BCGs considered for our statistical analysis are radio loud galaxies. Of the radio-loud population, $71 \%$ of the BCGs are located in re-

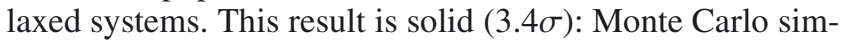
ulations show that the probability that this result is a chance detection is $\leq 3.4 \times 10^{-4}$. On the other hand, radio-quiet BCGs are mostly found in merging systems $(81 \%)$.

- We found that the fraction of radio BCGs in relaxed clusters increases with increasing value of the concentration parameter $c_{100}$, reaching $100 \%$ for $c_{100}>0.5$. For relaxed clusters $\left(c_{100}>0.2\right)$, we found a positive trend between $c_{100}$ and the $\mathrm{BCG}$ radio power, in the form $P_{1.4 \mathrm{GHz}} \propto c_{100}^{2.55 \pm 0.33}$. Since $c_{100}$ is an indicator of the cool-core strength $\left(c_{100} \propto t_{\text {cool }}^{-1}\right.$, e.g. Santos et al. 2008), this trend suggests that the most powerful BCGs are located in the strongest cool-core clusters, indicating a clear connection between the AGN activity of the BCGs and the deposition of the cooling gas at the cluster centre (see also Mittal et al. 2009).

- For the BCGs in relaxed clusters, there is only a weak correlation between the radio power of the BCGs and the core $\mathrm{X}$-ray luminosity of the host cluster (within $0.15 R_{500}$ ).

- The fractional radio luminosity function differs for the BCGs in the two environments. In particular, the BCGs in relaxed clusters have an extremely high probability to be radio loud, that is, almost $90 \%$, to be compared to the $\sim 20-30 \%$ for those in relaxed clusters.

- For a subset of our full sample ( 41\%), optical spectra are available in the SDSS. Eleven out of 28 of these spectra show emission lines, and these are all radio-loud BCGs in relaxed clusters, seven of them with a radio mini-halo.

Our study provides support for a strong link between the radio properties of BCGs and the dynamical state of the host cluster. We propose that our results reflect the AGN accretion mode of the BCGs. At least a fraction of the radio-loud BCGs in relaxed clusters may be accreting cold gas from the central region of the host cool-core cluster. This cold gas is certainly available for radio galaxies in mini-halo clusters, as their optical spectra show, and it is most likely less abundant in BCGs in merging clusters, where the dominant accretion mode for the radio AGN may be due to accretion of hot gas from the IGM of the galaxy itself. Understanding how the cold gas in the central cluster regions is transported all the way through the galaxy in the nearest proximity of the black hole remains an open issue.

Acknowledgements. We thank R. Fanti for the many insightful discussions. Thanks are due to S. Ettori for providing the routines we used for the Chandra $\mathrm{X}$-ray data analysis and for the derivation of the cluster morphological parameters. R.K. and T.V. acknowledge partial support by PRIN-INAF 2008 and by FP7-People-2009 IRSES CAFEGroups project under grant agreement 247653. Funding for SDSS-III has been provided by the Alfred P. Sloan Foundation, the Participating Institutions, the National Science Foundation, and the US Department of Energy Office of Science. The SDSS-III web site is http://www . sdss3.org/. SDSS-III is managed by the Astrophysical Research Consortium for the Participating Institutions of the SDSS-III Collaboration including the University of Arizona, the Brazilian Participation Group, Brookhaven National Laboratory, Carnegie Mellon University, University of Florida, the French Participation Group, the German Participation Group, Harvard University, the Instituto de Astrofisica de Canarias, the Michigan State/Notre Dame/JINA Participation Group, Johns Hopkins University, Lawrence Berkeley National Laboratory, Max Planck Institute for Astrophysics, Max Planck Institute for Extraterrestrial Physics, New Mexico State University, New York University, Ohio State University, Pennsylvania State University, University of Portsmouth, Princeton University, the Spanish Participation Group, University of Tokyo, University of Utah, Vanderbilt University, University of Virginia, University of Washington, and Yale University. This research has made use of the NASA/IPAC Extragalactic Database (NED) which is operated by the Jet Propulsion Laboratory, California Institute of Technology, under contract with the National Aeronautics and Space Administration.

\section{References}

Ahn, C. P., Alexandroff, R., Allende P. C., et al. 2014, ApJS, 211, 17 Auriemma, C., Perola, G. C., Ekers, R. D., et al. 1977, A\&A, 57, 41 Bacchi, M., Feretti, L., Giovannini, G., et al. 2003, A\&A, 400, 465 Balmaverde, B., Baldi R. D., \& Capetti, A. 2008, A\&A, 486, 119 Bardelli, S., Schinnerer, E., Smolic, V., et al. 2010, A\&A, 511, A1 Best, P. N., von der Linden, A., Kauffmann, G., et al. 2007, MNRAS, 379, 894 Böhringer, H., Schuecker, P., Guzzo, L., et al. 2004, A\&A, 425, 367 Buote, D. A., \& Tsai, J. C. 1995, ApJ, 452, 522

Brunetti, G., \& Jones, T. W. 2014, Int. J. Mod. Phys., 23, 1430007

Brunetti, G., Giacintucci, S., Cassano, R., et al. 2008, Nature, 455, 944

Burns, J. O. 1998, Science, 280, 400

Cassano, R., Ettori, S., Giacintucci, S., et al. 2010, ApJ, 721, L82

Cassano, R., Ettori, S., Brunetti, G., et al. 2013, ApJ, 777, 141

Clarke, T. E., Sarazin, C. L., Blanton E. L., et al. 2005, ApJ, 625, 478

Clarke, T. E., Blanton, E. L., Sarazin, C. L., et al. 2009, ApJ, 697, 1481

de Gasperin, F., van Weeren, R. J., Brüggen, M., et al. 2014, MNRAS, 444, 3130 Donahue, M., Bruch, S., Wang, E., et al. 2010, ApJ, 715, 881

Dunn, R. J. H., \& Fabian, A. C. 2008, MNRAS, 385, 757

Ebeling, H., Edge, A.,C., Böhringer, H., et al. 1998, MNRAS, 301, 881 Ebeling, H., Edge, A. C., Allen, S. W., et al. 2000, MNRAS, 318, 333

Edge, A. C., Oonk, J. B. R., Mittal, R., et al. 2010, A\&A, 518, L47 Eilek, J. A., Burns, J. O., O’Dea, C. P., et al. 1984, ApJ, 278, 37 Fabian, A. C., Celotti, A., Blundell, K. M., et al. 2002, MNRAS, 331, 369 Fanaroff, B. L., \& Riley, J. M. 1974, MNRAS, 167, 31

Feretti, L., \& Venturi, T. 2002, in Merging Processes in Galaxy Clusters (Kluwer Academic Publishers), Astrophys. Space Sci. Lib., 272, 163

Feretti, L., Fusco-Femiano, R., Giovannini, G., et al. 2001, A\&A, 373, 106 
R. Kale et al.: Radio properties of BCGs in the extended GMRT cluster sample

Feretti, L., Schuecker, P., Böhringer, H, et al. 2005, A\&A, 444, 157 Giacintucci, S., Venturi, T., Bardelli, S., et al. 2006, New Astron., 11, 437 Giacintucci, S., Venturi, T., Brunetti, G., et al. 2009, A\&A, 505, 45

Giacintucci, S., O’Sullivan, E., Vrtilek, J., et al. 2011a, ApJ, 732, 95

Giacintucci, S., Markevitch, M., Brunetti, G., et al. 2011b, A\&A, 525, L10 Giacintucci, S., Dallacasa, D., Venturi, T., et al. 2011c, A\&A, 534, A57

Giacintucci, S., Markevitch, M., Venturi, T., et al. 2014, ApJ, 781, 9

Giles, P. A., Maughan, B. J., Birkinshaw, M., et al. 2012, MNRAS, 419, 503

Govoni, F., Feretti, L., Giovannini, G., et al. 2001, A\&A, 376, 803

Haarsma, D. B., Leisman, L., Donahue, M., et al. 2010, ApJ, 713, 1037

Hardcastle, M. J., Evans, D. A., \& Croston, J. H. 2007, MNRAS, 376, 1849

Heckman, T., \& Best, P. N. 2014, ARA\&A, 52, 589

Hoffer, A. S., Donahue, M., Hicks, A., et al. 2012, ApJS, 199, 23

Hudson, D. S., Mittal, T. Reiprich, T. H., et al. 2010, A\&A, 513, A37

Hummel, E. 1981, A\&A, 93, 93

Kale, R., Venturi, T., Giacintucci, S., et al. 2013, A\&A, 557, A99

Kale, R., Venturi, T., Giacintucci S., et al. 2015, A\&A, 579, A92

Klein, U., Mack, K.-H., Gregorini, L., et al. 1995, A\&A, 303, 427

Ledlow, M. J., \& Owen, F. N. 1996, AJ, 112, 9

Lin, Y.-T., Brodwin, M., Gonzales, A. H., et al. 2013, ApJ, 771, 61

Liu, F. S., Mao, S., \& Meng, X. M. 2012, MNRAS, 423, 422

Macario, G., Venturi, T., Brunetti, G., et al. 2010, A\&A, 517, A43

Mahdavi, A., Hoekstra, H., Babul, A., et al. 2007, ApJ, 668, 806

Mauch, T., \& Sadler, E. M. 2007, MNRAS, 375, 931

Maurogordato, S., Cappi, A., Ferrari, C., et al. 2008, A\&A, 481, 593
Mazzotta, P., \& Giacintucci, S. 2008, ApJ, 675, L9

Mazzotta, P., Bourdin, H., Giacintucci, S., et al. 2011, Mem. It. Astron. Soc., 82,495

McNamara, B. R., \& Nulsen, P. E. J. 2007, ARA\&A, 45, 117

McNamara, B. R., \& Nulsen, P. E. J. 2012, New J. Phys., 14, 5023

Mittal, R., Hudson, D. S., Reiprich, T. H., et al. 2009, A\&A, 501, 835

Murgia, M., Govoni, F., Markevitch, M., et al. 2009, A\&A, 499, 679

O’Dea, C. P., Baum, S. A., Privon, G., et al. 2008, ApJ, 681, 1035

O'Dea, K. P., Quillen, A. C., O'dea, C. P., et al. 2010, ApJ, 719, 1619

Ogrean, G. A., Hatch, N. A., Simionescu, A., et al. 2010, MNRAS, 406, 354

Orrú, E., Murgia, M., Feretti, L., et al. 2007, A\&A, 467, 943

Owen, F. N., \& Laing, R. A. 1989, MNRAS, 238, 357

Peterson, J., \& Fabian, A. C. 2006, Phys. Rev., 407, 1

Poole, G. B., Faradal, M. A., Babul, A., et al. 2006, MNRAS, 373, 881

Quintana, H., \& Lawrie, D. G. 1982, AJ, 87, 1

Reiprich, T. H., \& Böhringer, H. 2002, ApJ, 567, 716

Santos, J. S., Rosati, P., Tozzi, P. et al. 2008, A\&A, 483, 35

Sun, M. 2009, ApJ, 704, 1586

Valtchanov, I., Murphy, T., \& Pierre, M. 2002, A\&A, 392, 795

Venturi, T., Giacintucci, S., Brunetti, G., et al. 2007, A\&A, 463, 937

Venturi, T., Giacintucci, S., Dallacasa, D., et al. 2008, A\&A, 484, 327

Venturi, T., Giacintucci, S., Dallacasa, D., et al. 2011, MNRAS, 414, L65

Venturi, T., Giacintucci, S., Dallacasa, D., et al. 2013, A\&A, 551, A24

ZuHone, J. A., Markevitch, M., Brunetti, G., et al. 2013, ApJ, 762, 78 